\title{
Long Segment Spinal Cord Infarction after Thoracic Endovascular Aortic Repair Implementation
}

\author{
Sungjoon Yoon, $\mathrm{MD}^{*}$ (D); Seon-Kyung Lee, $\mathrm{MD}^{\dagger}$ (D); Hyung-Min Kwon, $\mathrm{MD}, \mathrm{PhD}^{*}$ (D) \\ Department of Neurology, SMG-SNU Boramae Medical Center, Seoul National University College of Medicine*, Seoul; Department of \\ Neurology, Seoul National University College of Medicine ${ }^{\dagger}$, Seoul, Korea
}

The clinical manifestation of spinal cord infarction (SCI) varies according to the involved vascular territory. Aortic disease is the most common cause. Most importantly, SCI after thoracic endovascular aortic repair (TEVAR) occasionally occurs. During surgery, embolism caused by mural hematoma can cause multiple territory infarctions in the spinal cord, brainstem, and cerebellum. In almost all cases, the lesion was focal, but this case showed long segment SCI (thoracic vertebral level 1 to conus medullaris). This patient's initial symptom was paraplegia, but the symptoms were aggravated by quadriplegia and respiratory depression. As long segment SCI is uncommon, we present a valuable case of SCI after TEVAR implementation.

\section{J Neurosonol Neuroimag 2020;12(2):79-\$2}

Key Words: Spinal cord ischemia; Endovascular procedure; Thromboembolism
Received: September 21, 2020

Revised: November 12, 2020

Accepted: November 12, 2020

Correspondence:

Hyung-Min Kwon, MD, $\mathrm{PhD}$

Department of Neurology, SMG-SNU Boramae Medical Center, Seoul National University College of Medicine, 20 Boramae-ro 5-gil, Dongjak-gu, Seoul 07061, Korea

Tel: $+82-2-870-2475$

Fax: $+82-2-831-2826$

E-mail: hmkwon@snu.ac.kr
Spinal cord infarction (SCI) is a disease with a low prevalence rate accounting for $0.3-1.0 \%$ of all strokes. SCI should be considered a cause for various clinical symptoms and signs such as motor and sensory symptoms, respiratory depression, and autonomic dysfunction. ${ }^{1}$ The causes of SCI vary widely. Above all, the common causes are aortic disease with traumatic or iatrogenic etiology and thrombotic occlusion. ${ }^{2}$ When compared to open transabdominal aortic aneurysm repair procedures, the SCI occurrence rate showed no significant difference in thoracic endovascular aortic repair (TEVAR). However, the occurrence of delayed onset SCI was greater in TEVAR than in open thoracoabdominal aortic aneurysm repair. ${ }^{3}$ Postoperative SCI is an important prognostic factor. The mortality rate is three times higher in patients with SCI than in those without SCI. ${ }^{3}$

Therefore, it is very important for surgeons and neurologists to keep in mind that SCI may arise from complications of aortic repair surgery or procedure. Here, we present a case of long segment SCI as a TEVAR complication.

\section{CASE}

A 74-year-old man presented to the emergency department with chest pain that seemed to tighten both sides and radiated pain that stretched over his shoulder, which started 2 days before the hospitalization. He was on aspirin and cilostazol for three-vessel disease of the coronary artery. The patient had been previously diagnosed with atrial fibrillation, hypertension, and benign prostate hypertrophy. On coronary angiography, there was up to $90 \%$ stenosis with an ulcerative plaque in the left anterior descending coronary artery. Diffuse aortic aneurysm was found on computed tomography (CT) angiography of the thoracic and abdominal aorta. The aortic aneurysm extended from the ascending aorta, aortic arch, and descending thoracic aorta (Fig. 1) with a mural thrombus and a maximum diameter of 6.3 $\mathrm{cm}$ in the aortic arch, which is an indication for hybrid TEVAR. ${ }^{4}$ Therefore, elective surgery for thoracic aortic aneurysm was scheduled. The patient subsequently underwent on-pump beating-heart coronary artery bypass grafting and modified type II hybrid arch repair. 
The duration of the surgery was 13 hours 35 minutes in total, and mean blood pressure was maintained above $60 \mathrm{mmHg}$ with invasive arterial blood pressure. The patient was unconscious until the second day after surgery and underwent brain CT, which showed no apparent lesion. The patient's consciousness improved on the fourth day after surgery, but he was referred to a neurologist for paraplegia. On neurological examination, his consciousness was intact, but the patient was intubated, and his lower extremities showed more severe weakness (Medical Research Council [MRC] grade $\mathrm{O}$ on both sides) than the upper extremity (MRC grade 4 on both sides). Spine magnetic resonance imaging performed to rule out SCI revealed a wide range of restricted diffusion in the spinal cord from the thoracic vertebrae 1 level to the conus medullaris with T2 hyperintensity (Fig. 2). Dual antiplatelet (aspirin 100 mg daily, clopidogrel $75 \mathrm{mg}$ daily) therapy was administered as secondary prevention. The patient was in the process of ventilator weaning, but on the fifth day after the surgery, failure of weaning and carbon dioxide $\left(\mathrm{CO}_{2}\right)$ retention (partial pressure of $\mathrm{CO}_{2}$ ranging from 43.3 to $48.3 \mathrm{mmHg}$ ) were observed. At the same time, weak- ness of both upper extremities progressed from MRC grade 4 to MRC grade 2 on both sides. The possibility of a recurrent embolic infarction was considered at the level of the cervical cord and brainstem, but additional brain and spinal cord imaging were unavailable due to his critical condition. In addition, a CT scan of the abdomen and pelvis was performed due to elevation of the liver enzymes on the 4 th day after the surgery and showed the perfusion delay on segment three and segment six tips of the liver and spleen. Discoloration and delayed capillary refill time in both feet led to the diagnosis of blue toe syndrome on the second day after the surgery.

However, anticoagulation therapy was delayed due to the patient's poor bleeding control. The second surgery to explore and control the bleeding focus was performed 7 hours after the first surgery, in which no arterial bleeding was found. The hemoglobin level in complete blood count was $11.4 \mathrm{~g} / \mathrm{dL}$ initially but dropped to $8.9 \mathrm{~g} / \mathrm{dL}$ on the second day after the surgery.

Therefore, an additional $1 \mathrm{mg} / \mathrm{kg}$ enoxaparin daily, low molecular weight heparin after stabilization with massive transfusion of packed red blood cells, fresh
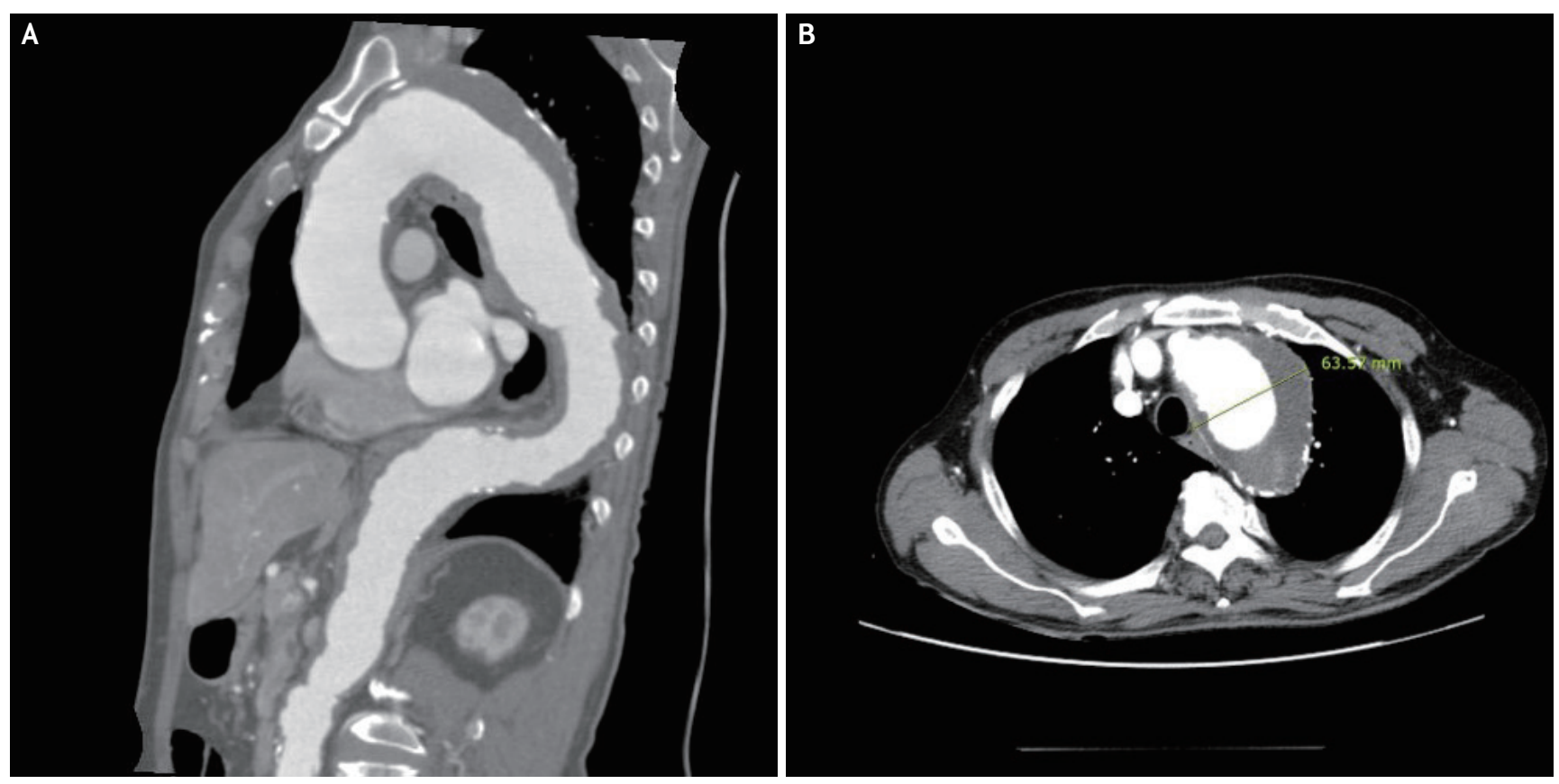

FIG. 1. Diffuse aortic aneurysm in computed tomography (CT) angiography of thoracic and abdominal aorta. (A) Diffuse aneurysm involving the ascending aorta, aortic arch and descending aorta with intramural thrombus and atherosclerotic calcification is observed in sagittal reformatted CT image. Diffuse atherosclerosis of the abdominal aorta is also seen. (B) Aortic aneurysm with large intramural thrombus at the aortic arch level (maximal diameter, $6.3 \mathrm{~cm}$ ) is noted in axial CT image. 
frozen plasma, platelet cells, and cryoprecipitate were administered. Afterward, acute respiratory distress syndrome and acute kidney injury aggravated; thus, continuous renal replacement therapy was performed. However, the patient did not recover and died due to the deterioration of metabolic acidosis and low blood pressure.

\section{DISCUSSION}

If a patient develops sudden back pain with tightness, symmetric flaccid weakness, and sensory loss, the neurologist should not overlook the possibility of SCI. These symptoms are common in SCI. If a patient has undergone aortic repair surgery or procedure recently, the possibility of SCI increases. Previously, the popular opinion was that damage to the artery of Adamkiewicz is the main cause of SCI during aortic aneurysm repair. ${ }^{5}$ Therefore, clinicians aimed to avoid injury of the left vertebral artery or internal iliac artery. ${ }^{2}$ However, a recent concept is that hypoperfusion and insufficient compensation from the collateral flow are two main contributors to SCI. ${ }^{6}$ Supporting that concept, previous studies showed that the number of segmental arteries (i.e., intercostal and lumbar arteries) sacrificed and hemodynamic instability are important risk factors for SCI. Recent techniques to reduce the risk of SCI (i.e., multistaged procedure, ${ }^{6}$ perioperative cerebrospinal fluid drainage, ${ }^{7}$ hypothermia during proximal artery clamping, ${ }^{8}$ embolization, and intraoperative neurophysiologic monitoring ${ }^{9}$ ) are based on these laboratory and clinical studies. Some of these techniques have led to positive results. ${ }^{6,710}$

The pathogenesis of SCI after TEVAR seems multifactorial. The hypothesis is that atheroembolism of aortic plaque material into the segmental arteries supplying the spinal cord can also lead to $\mathrm{SCI}^{2}$ In this patient, the evidence of postoperative atheroembolism was documented with blue toe syndrome and delayed arterial perfusion of the spleen, liver, and kidney in the abdominal and pelvic CT. Therefore, we can infer that
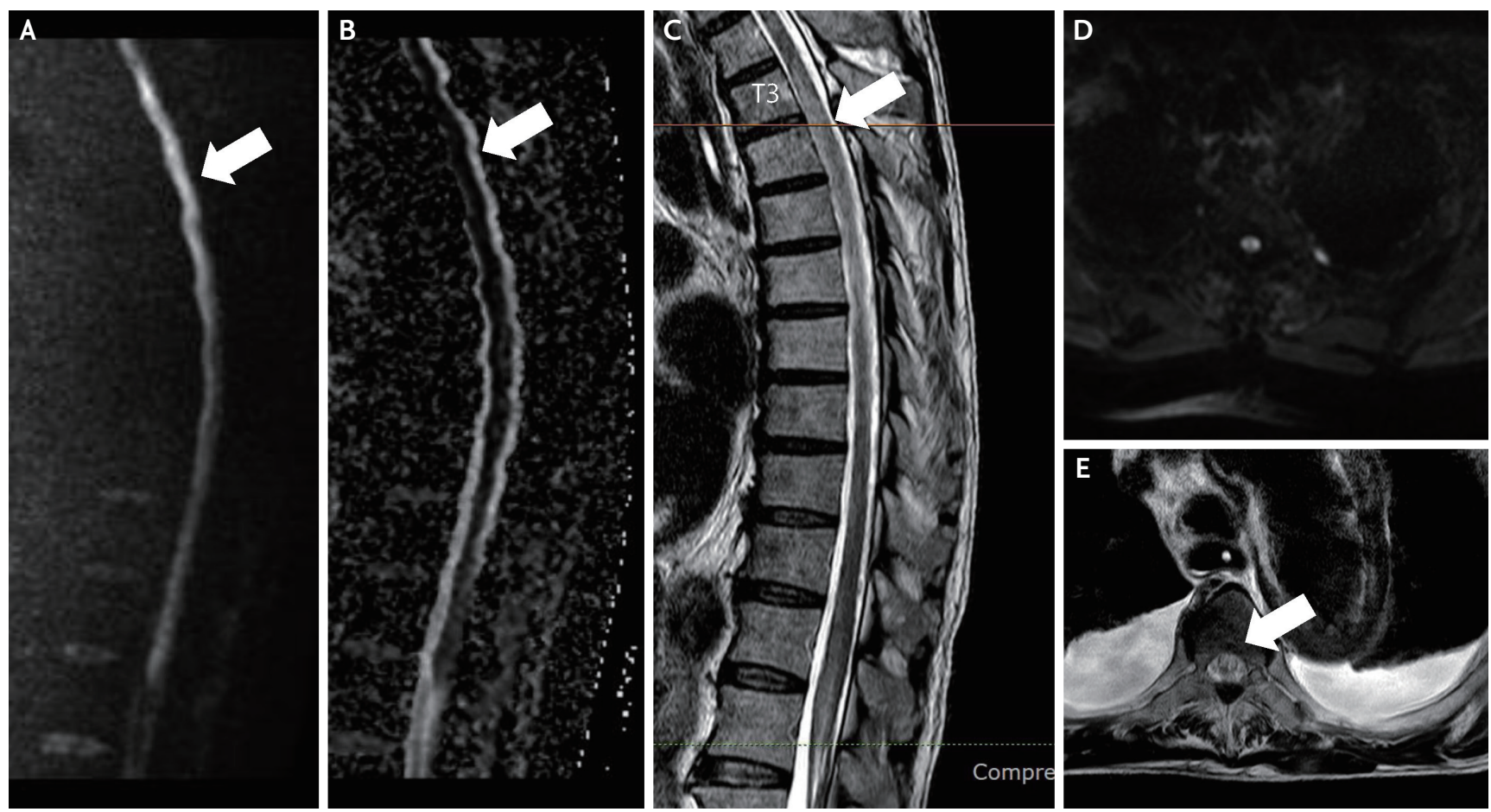

FIG. 2. Long segmental spinal cord infarction. (A) Sagittal diffusion weighted image, (B) sagittal ADC image, (C) sagittal T2-weighted image, (D) axial diffusion weighted image ( $T_{1}$ level), (E) axial $T_{2}$-weighted image ( $T_{3}$ level). Wide range of restricted diffusion (arrows of $A, B$ ) in the thoracolumbar spinal cord from $T_{1}$ level to conus medullaris is noted with high signal on T2-weighted image (arrows of C, E). Axial diffusion and $\mathrm{T}_{2}$-weighted images also demonstrate signal hyperintensity in the spinal cord. Bilateral symmetric $\mathrm{T}_{2}$ high signal intensity lesion sparing dorsal column are shown in axial $\mathrm{T}_{2}$-weighted image. 
mural thrombi embolization through the collateral network of blood vessels in the spinal cord, including the anterior spinal artery, contributed to the occurrence of SCI during and after TEVAR.

To a large extent, coverage of $\geq 2$ vascular territories and coverage including the left subclavian artery are known surgical risk factors for SCI after TEVAR, ${ }^{5}$ which also explains this case. This case is a good example of long segment SCI with mural thrombi embolization after TEVAR.

\section{Conflicts of Interest}

No potential conflicts of interest relevant to this article was reported.

\section{REFERENCES}

1. Romi F, Naess H. Spinal cord infarction in clinical neurology: a review of characteristics and long-term prognosis in comparison to cerebral infarction. Eur Neurol. 2016;76:95-98.

2. Cheshire WP, Santos CC, Massey EW, Howard JF Jr. Spinal cord infarction: etiology and outcome. Neurology. 1996;47:321-330.

3. Maeda T, Yoshitani K, Sato S, Matsuda H, Inatomi Y, Tomita Y, et al. Spinal cord ischemia after endovascular aortic repair versus open surgical repair for descending thoracic and thoracoabdominal aortic aneurism. J Anesth. 2012;26:805-811.

4. Grabenwöger M, Alfonso F, Bachet J, Bonser R, Czerny M,
Eggebrecht H, et al. Thoracic endovascular aortic repair (TEVAR) for the treatment of aortic diseases: a position statement from the European Association for Cardio-Thoracic Surgery (EACTS) and the European Society of Cardiology (ESC), in collaboration with the European Association of Percutaneous Cardiovascular Interventions (EAPCI). Eur Heart J. 2012;33:1558-1563.

5. Awad H, Ramadan ME, El Sayed HF, Tolpin DA, Tili E, Collard CD. Spinal cord injury after thoracic endovascular aortic aneurysm repair. Can J Anaesth. 2017;64:1218-1235.

6. Etz CD, Zoli S, Mueller CS, Bodian CA, Di Luozzo G, Lazala $\mathrm{R}$, et al. Staged repair significantly reduces paraplegia rate after extensive thoracoabdominal aortic aneurysm repair. J Thorac Cardiovasc Surg. 2010;139:1464-1472.

7. Coselli JS, LeMaire SA, Köksoy C, Schmittling ZC, Curling PE. Cerebrospinal fluid drainage reduces paraplegia after thoracoabdominal aortic aneurysm repair: results of a randomized clinical trial. J Vasc Surg. 2002;35:631-639.

8. Etz CD, Homann TM, Plestis KA, Zhang N, Luehr M, Weisz DJ, et al. Spinal cord perfusion after extensive segmental artery sacrifice: can paraplegia be prevented? Eur J Cardiothorac Surg. 2007;31:643-648.

9. Husain AM, Swaminathan M, McCann RL, Hughes GC. Neurophysiologic intraoperative monitoring during endovascular stent graft repair of the descending thoracic aorta. J Clin Neurophysiol. 2007;24:328-335.

10. Griepp RB, Griepp EB. Spinal cord protection in surgical and endovascular repair of thoracoabdominal aortic disease. J Thorac Cardiovasc Surg. 2015;149 Suppl 2:S86-S9o. 